\title{
Characterization of Si in a W matrix Using Diffraction Contrast in the TEM
}

\author{
B.I. Prenitzer, B.W. Kempshall, ${ }^{* *}$ J.M. McKinley, W.H. Stinebaugh, ${ }^{* * *}$ and I. Wylie ${ }^{* * * *}$ \\ *Agere Systems 9333 S. John Young Parkway, Orlando, FL 32819 \\ ** Mechanical, Materials and Aerospace Eng., University of Central Florida, Orlando, FL 32816 \\ ***A Agere Systems 600-700 Mountain Avenue, PO Box 636, Murray Hill, NJ 07974 \\ Agere Systems 2 Oak Way, Berkeley Heights, NJ 07922
}

The practice of using a $\mathrm{Si}$ based gas prior to $\mathrm{W}$ deposition is a process used within the semiconductor industry to aid in uniform and continuous filling of interlevel interconnects. SIMS has been effectively used to determine the concentration of $\mathrm{Si}$ in the bulk of a $\mathrm{W}$ nucleation layer formed during a blanket deposition. RBS has also been employed to evaluate the W:Si ratio in the same nucleation layer. Such techniques have proven effective in the detection of low levels of Si in $\mathrm{W}$; however, the lateral spatial resolution of these techniques is orders of magnitude larger than the dimensions necessary to directly analyze modern integrated circuit devices. Furthermore, it is postulated that the material character observed in films produced by blanket deposition may have subtle but significant differences from the material deposited within the confines of a high aspect ratio via (i.e., $350-500 \mathrm{~nm}$ in diameter $\mathrm{x} 1000 \mathrm{~nm}$ deep.) Therefore, the TEM becomes the instrument of choice for the site-specific analytical characterization of such features. Although TEM is unquestionably capable of the requisite lateral spatial resolution, the analysis, more specifically the spectroscopic analysis, remains non-trivial. X-ray energy dispersive spectrometry (XEDS) techniques are severely hampered by the fact that the typical energy resolution of an XEDS detector is not sufficient to resolve the characteristic X-ray energy levels between the $\mathrm{Si} \mathrm{K} \alpha(1.737 \mathrm{keV})$ and the $\mathrm{W} \mathrm{M} \alpha(1.774 \mathrm{keV})[1,2]$. Although the energy resolution of wavelength dispersive X-ray spectrometry (WDS) is sufficient to detect $\mathrm{Si}$, the lateral spatial resolution can be problematic and more significantly, X-ray fluorescence from the surrounding $\mathrm{SiO}_{2}$ matrix obscures the analysis. Electron energy loss techniques also are capable of the requisite energy resolution, but when the specimen is thinned sufficiently for the analysis the surrounding $\mathrm{SiO}_{2}$ matrix has been observed to become mechanically unstable under the electron beam. Lastly, the microcalorimeter, which is a seemingly viable option, has not yet achieved widespread availability and may be subject to the same fluorescence artifacts as observed in WDS.

By exploiting fundamental diffraction contrast mechanisms that are readily available in conventional TEM, a method to detect the presence of $\mathrm{Si}$ in $\mathrm{W}$ has been developed. The effect has been observed in both TEM and STEM modes of operation using both a Philips EM430 operating with a LaB 6 source at $300 \mathrm{kV}$ as well as a Philips/FEI Tecnai F30 operating with a field emission gun at $300 \mathrm{kV}$. In blanket films deposited using the $\mathrm{Si}$ gas process a distinct bright band of $\sim 4 \mathrm{~nm}$ is observed in dark field (DF) TEM images which are formed by allowing diffracted beams that correspond to lattice spacings less than $0.100 \mathrm{~nm}$ to pass through the objective aperture. Any such band is systematically absent in analogously formed DF images of blanket films deposited without the introduction of the $\mathrm{Si}$ gas as shown in Figure 1. Figures $2 \mathrm{a}$ and $2 \mathrm{~b}$ show corresponding bright field (BF) and DF TEM images, respectively, of a fully processed $\mathrm{W}$ via. The site-specific plan view specimen was prepared using the in-situ FIB lift-out technique[3]. Figure 2b, clearly shows a bright band $\sim 5 \mathrm{~nm}$ thick. The initial Si gas stage of the deposition process has the potential to create a region of relatively high $\mathrm{Si}$ concentration prior to the introduction of W. TEM analysis suggests that the excess Si may be consumed in a reaction with $\mathrm{W}$ at the perimeter of the $\mathrm{W}$ plug to form a $\mathrm{W}_{\mathrm{x}} \mathrm{Si}_{\mathrm{y}}$ phase that is detectable by diffraction contrast mechanisms. Identification of constituents contributing to the 
bright band has been made based on X-ray data published in the JCPDS[4]. The $\mathrm{WSi}_{2}$ phase alone has at least 16 reflectors with a normalized intensity greater than 10 that could potentially contribute to the DF image under the prescribed imaging conditions. Although $\mathrm{Ti}, \mathrm{TiN}, \mathrm{W}$, and $\mathrm{Si}$ all generate reflectors that could contribute to the aforementioned DF image, both the number and the relative intensities of the $\mathrm{WSi}_{2}$ reflectors are at least 4 times greater than those of the other candidates. Furthermore, the location of this band (i.e., it is completely contained within the $\mathrm{W}$ and clearly not an interfacial layer) corresponds to where the formation of such a silicide phase would be anticipated. Thus, the empirical evidence strongly suggests that dark field imaging in conventional TEM mode can be used to detect the presence of Si in W.

\section{References}

[1] D.B. Williams and C.B.Carter Transmission Electron Microscopy, Plenum, New york, 1996

[2] J.I. Goldstein et al., Scanning Electron Microscopy and X-ray Microanalysis, Plenum, NewYork, 1992

[3] T. Kamino et al., Microsc. Microanal. 6 (Suppl. 2) (2000) 501

[4] Joint Committee on Powder Diffraction Standards, Swarthmore, PA 19081 The support of Omniprobe is greatly appreciated
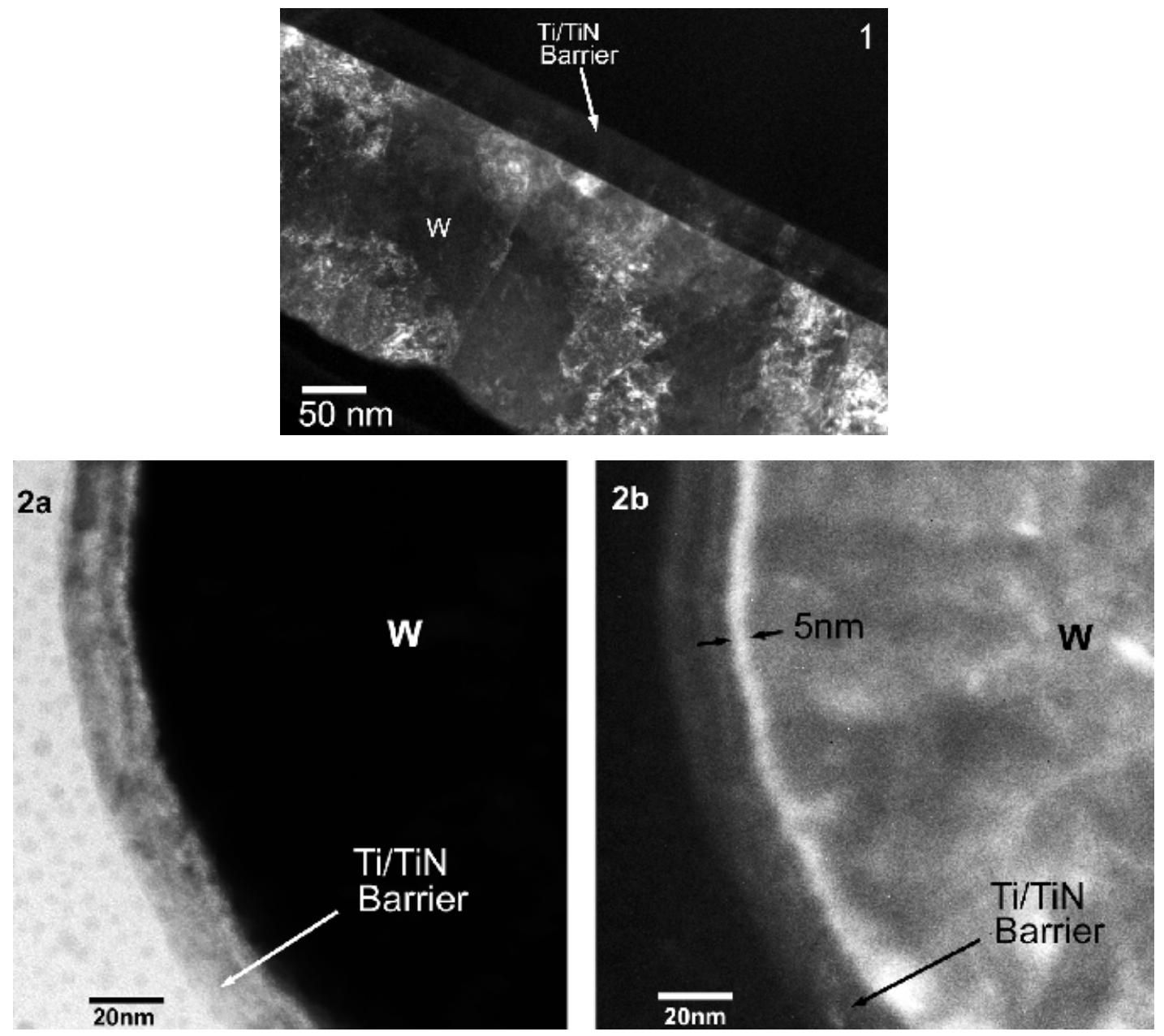

Figure 1. DF TEM image showing a W layer deposited by CVD without the use of the Si gas process.

Figure 2a. BF TEM image of a fully processed $\mathrm{W}$ plug in which the Si gas process was used.

Figure 2b. Corresponding DF TEM image of a W plug showing the bright band indicative of the presence of $\mathrm{W}_{\mathrm{x}} \mathrm{Si}_{\mathrm{y}}$. 